\title{
Improvement of One-Cycle Controller Response with a Current Mode Controller
}

\author{
Mohsen Ruzbehani ${ }^{\dagger}$, Luowei Zhou*, and Nasser Mirzaei** \\ $\dagger * *$ Laser \& Optics Research School, NSTRI, Tehran, Iran \\ ${ }^{*}$ College of Electrical Engineering, Chongqing University, Chongqing, P.R. China
}

\begin{abstract}
The most important feature of the one-cycle control method is its excellent ability in line disturbance rejection. However, when it is used as a controller in dc-dc converters, it has an undesirable transient response. The voltage overshoot at the transient time, which usually exists in one-cycle controlled converters, is unwanted in many applications and it is sometimes hazardous. In this paper, it is shown that the combination of a one-cycle controller with a current mode controller, can improve the transient response and consequently the overshoot can be controlled. Therefore, the combined controller has the excellent line disturbance rejection of a one-cycle controller and the output current limiting capability of current mode controllers. Because in this scheme a one-cycle controller is the master controller, the problem of instability of current mode control, will not happen. By simulation and a practical prototype, the capability of the method is shown.
\end{abstract}

Key Words: Current limiter, Current mode control, One-cycle control, Power converter

\section{INTRODUCTION}

The main idea of One-Cycle Control (OCC) is to use an integrator to measure the mean value of the diode signal in each cycle and force it to be equal to the reference value. By this simple mechanism the input signal to the low pass filter, is controlled so that its average value, i.e. the output of the filter, is equal to the reference signal [1]. A decade after the invention of OCC, it has found applications in several areas of power electronics, such as APF and PFC, control of dcdc converters, switching amplifiers, etc [1]-[3]. Even recently, an integrated one-cycle controlled converter has been reported [4].

Another method, which has been used for decades in power converter control, is Current Mode Control (CMC). Its main advantages over duty ratio programming, such as better linenoise reduction, automatic overload protection, effective first order transfer function for buck converters and the fact that it is easy to parallel by using a common control, has made it a very popular controll method [5]-[8].

There are two main approaches in $\mathrm{CMC}$, namely Peak Current Mode (PCM) and Average Current Mode (ACM). In PCM cycle by cycle peak current limiting is possible. However, its drawbacks are its open-loop instability and sub harmonic oscillations. The instability problems can be solved

\footnotetext{
Manuscript received Aug. 30, 2009; revised Nov. 25, 2009

$\dagger$ Corresponding Author: mruzbehani@aeoi.org.ir

Tel: +98-21-82064105, Fax: +98-21-88221083, Laser \& optics research school

* College of Electrical Engineering, Chongqing University, P.R. China

** Laser \& optics research school, Iran
}

by means of an artificial ramp signal either subtracted from the control signal or added to the inductor-current signal [5], [6]. Unfortunatly, due to the variation of conditions, this method is sometimes difficult to apply and it also degrades the advantages of PCM. Generally speaking in PCM, noise sensitivity and the differences between peak and average current values, especially in high power factor boost pre regulators, are the main problems [7].

In this paper a combination of OCC and PCM is proposed. It is shown that this combination improves the transient behavior of OCC. Furthermore, by using CMC, the switch stress can be reduced and the overload protection characteristic of CMC is added to the features of OCC. It should be noted that because in this method the PCM only works in transient time, it is not necessary to consider the common instability problem of this controller. This method also simplifies the implementation of one cycle controlled boost converters.

In section 2, a review of the OCC method for buck and boost converters is given. In section 3, by studying the effects of a current limiter on the transient response of the converter, the idea of this paper is introduced. More discussion about this method and simulation results are given in section 4 . The practical verification of the simulation results is the subject of section 5 .

\section{ONE-CYCLE CONTROL OF BUCK AND BOOST CONVERTERS}

In this section one-cycle control of buck and boost converters is described. The basic schemes which are used, and the 


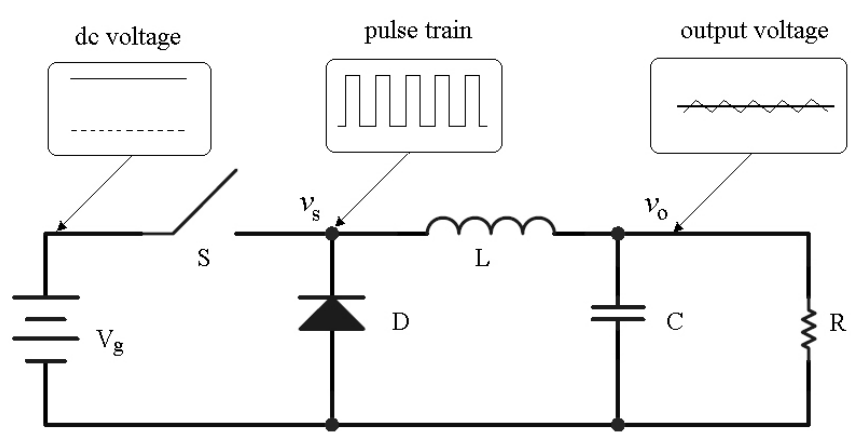

Fig. 1. Buck converter and its nodes waveforms.

operation of the controllers for these two topologies, are the subjects of this section.

\section{A. Buck converters}

The main feature of OCC in converter control is its ability to cancel out the effects of line voltage fluctuations without output feedback in one cycle. In fact OCC blocks line disturbance from the output before it can appear in the output. On the other hand, in conventional feedback with PID control, the effects of line disturbance appear in the output before the controller can act, no matter how fast this procedure is done.

The basic concept of OCC can be understood by considering buck converter operation. In these converters, it is possible to control the average value of the input signal to the low-pass filter by changing the switch duty cycle. Consequently, the average value of the output signal can be controlled. Hence, if we control the average value of the pulse train which is applied to the filter in each cycle, it is possible to control the dc value of the output voltage. In Fig. 1 a buck converter with its nodes' waveforms has been sketched. At the input of the filter there is a pulse train with a magnitude that is equal to the input voltage and its average is determined by the following relation [1]-[8].

$$
V_{s}=\frac{1}{T_{s}} \int_{0}^{T_{s}} v_{s} d t=\frac{1}{T_{s}} \int_{0}^{d T_{s}} v_{g} d t=d v_{g}
$$

where, $\mathrm{d}$ is the duty cycle and $T_{s}$ is the sampling period. Integrating $v_{s}$ and changing the duty cycle in a manner such that the integral is equal to a predefined reference, is the core of the one-cycle control method. Therefore, the control law of one-cycle control of buck converters can be defined as follows:

$$
v_{r e f}=\frac{1}{T_{s}} \int_{0}^{d T_{s}} v_{g} d t
$$

With this method, it is possible to remove the disturbance in the line voltage in one cycle.

Despite this, it is known that one-cycle controllers do not have a good step response. In the conventional PWM method at the transient time, due to the magnitude of the error signal, its derivative and its integral, the duty cycle is changed so that a desirable result is obtained. However, in the OCC method, the amplitude of the error signal has no effect on the duty

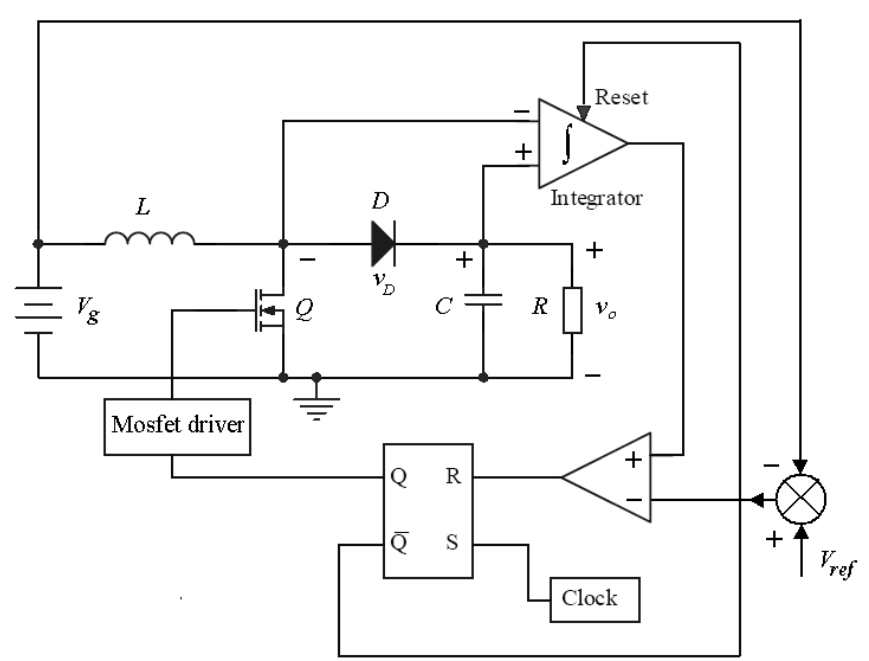

Fig. 2. Scheme of one-cycle controlled boost converter.

cycle. Therefore, the transient response is not controllable. The condition is worse when the load and filter act as an under damped system. In this case, the output signal oscillates too much before reaching the steady state. It is well known that output voltage overshoot in some applications such as microprocessor power supplies is not acceptable. Therefore, especially in those applications, using a conventional onecycle controller without any improvement is not possible. One way to deal with this problem is to use an auxiliary PID or a nonlinear controller [9], [10]. The other method, which will be discussed in this paper, is to use a current control in combination with OCC.

\section{B. Boost converters}

For buck converters the integral of the diode voltage is used as a measure for the operation of the controller. In boost converters, it is possible to compare the integral of the diode voltage with a reference signal as is shown in Fig. 2. The control law in this case is as follows [8]:

$$
v_{r e f}-V_{g}=\frac{1}{T_{s}} \int_{0}^{d T_{s}} v_{D} d t=v_{0} d
$$

Fig. 2 shows the general idea. In practice this circuit cannot start from the zero condition. At the beginning when the output voltage and the diode voltage are zero, the integral of this voltage will never exceed the value of the reference and consequently the switch is always on. To solve this problem in the implementation of a one-cycle controller for boost converters, the output of the RS flip-flop should be ANDed with a square wave with a maximum practical duty cycle. Therefore at the beginning when the diode voltage is less than the reference voltage, the switch works with a maximum dutycycle. It can be seen that in OCC of a buck converter, when there is no line variation, the duty cycles at the transient and steady state are the same. This is not the case in one-cycle controlled boost converters.

However, because in both cases it is the output voltage which limits the inductor current, at transient time when the 


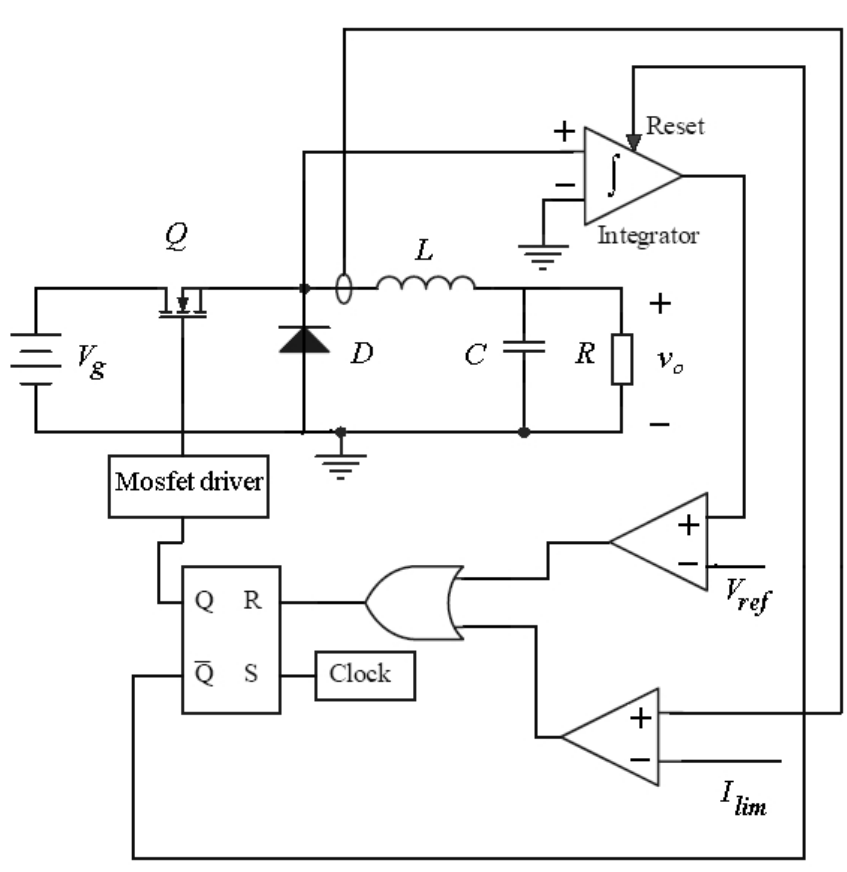

Fig. 3. Improved scheme of one-cycle control of buck converter by use of current limiter.

output voltage is lower than the input, the inductor current has an overshoot. The overshoot of the inductor current influences the transient behavior of the output voltage; consequently, as will be shown in next section, by eliminating the inductor current, the transient response of the controller can be improved.

\section{EFFECT OF A CURRENT LIMITER ON THE OPERATION OF ONE-CYCLE CONTROLLERS}

The overshoot of the inductor current at the transient time influences the transient behavior of the output voltage. In this section, it will be shown that by limiting the inductor current it is possible to improve this effect. A buck converter is shown in Fig. 3. As can be seen, the reset command of the flip-flop that determines the duty cycle is governed by two factors. The first factor is the exceeding of the integral of the diode voltage from the reference voltage. The second factor is the exceeding of the inductor current above the current limit. Therefore, at the transient time or in the case of a load change, when the inductor current has the tendency to increase, this is the current control part which governs the duty cycle. However, at the steady state the one-cycle controller does. For the correct operation of a controller an $I_{\text {Lim }}$ should be chosen that is greater than the steady state value of the inductor control, otherwise the controller will act as an ordinary current mode controller and the one-cycle controller will be disabled.

By this method, the transient response of OCC can be improved. On the other hand, in the steady state the controller acts as an ordinary OCC with its essential advantage of line disturbance reduction.

In Fig. 4, a scheme of the current limited OCC method for boost converters has been shown. The idea is the same as for buck converters. The other benefit of this method in boost converters is the effect of a current limiter on the start

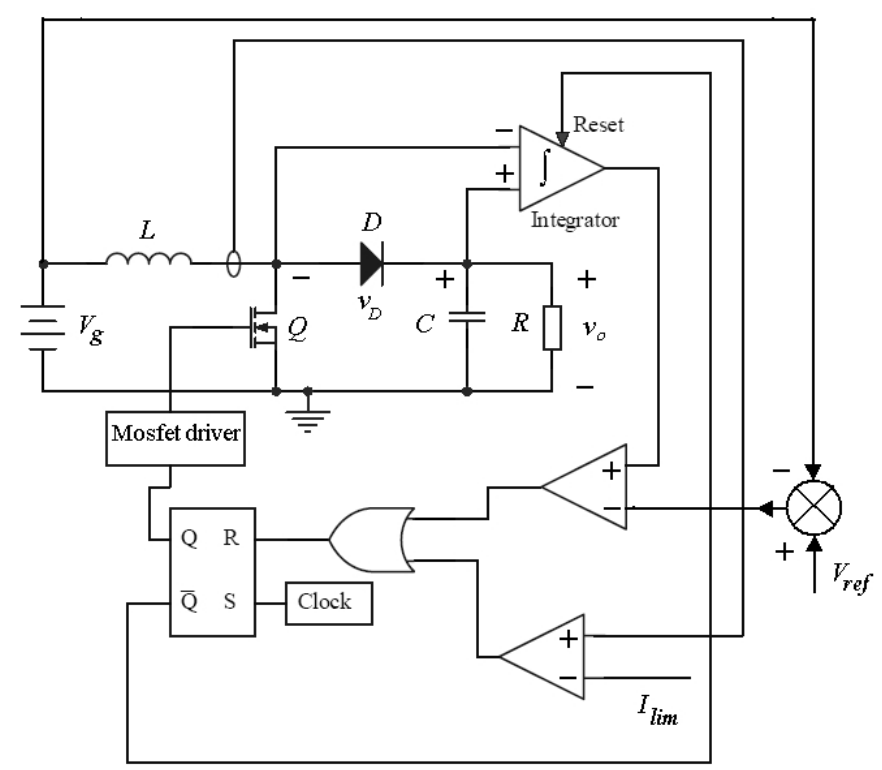

Fig. 4. Improved scheme of one-cycle control of boost converter by use of current limiter.

up of the converter. As described previously, an ordinary OCC for a boost converter needs an extra circuit for start up and it cannot start from the zero condition. However, in the combined method, the current limiter part determines the duty cycle so the previously mentioned problem does not occur.

\section{Simulation Results}

In this section, the simulation results of the current limited one-cycle control method for buck and boost converters will be given. The simulations have been done with the Saber program. For the first simulation, a buck converter with the following parameter has been considered.

As expected the one-cycle control eliminated the line variation completely. Furthermore as shown in Fig. 5, by changing the current limit value, the transient behavior of the output voltage changes from oscillatory to damped behavior. By reducing the current limit, the magnitude of the overshot of the output voltage is reduced. In Fig. 6, the effect of the current limit on the inductor current is shown. As can be seen, by limiting the value of the current limit the oscillatory behavior of the inductor current is reduced. The current limiter effect is considerable when the inductor current overshoot is so large that the converter goes into the discontinuous conduction mode for some moments. This matter is shown in the boost converter simulations.

In the next simulation, a boost converter is examined. In this example the parameter are as follows:

$L=200 \mu H, r_{L}=0.1 \Omega, C=500 \mu F, R=10 \Omega, V_{g}=$ $20 \mathrm{~V}, V_{0}=50 \mathrm{~V}$ and $f=20 \mathrm{kHz}$.

Again, the effect of the current limiter on the transient behavior of the converter is simulated. As shown in Fig. 7, by decreasing the value of the current limit, the magnitude of the oscillations of the output voltage are decreased. In Fig. 8, the inductor current is shown. In this simulation the current overshoot is too large, so the converter goes into discontinuous conduction mode for some milliseconds. 


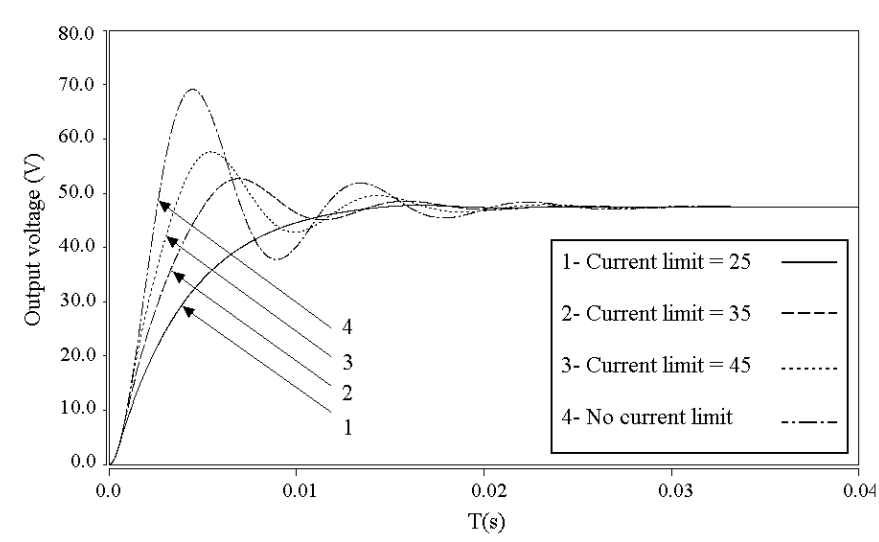

Fig. 5. Simulation results of output voltage of a buck converter due to various values of current limit.

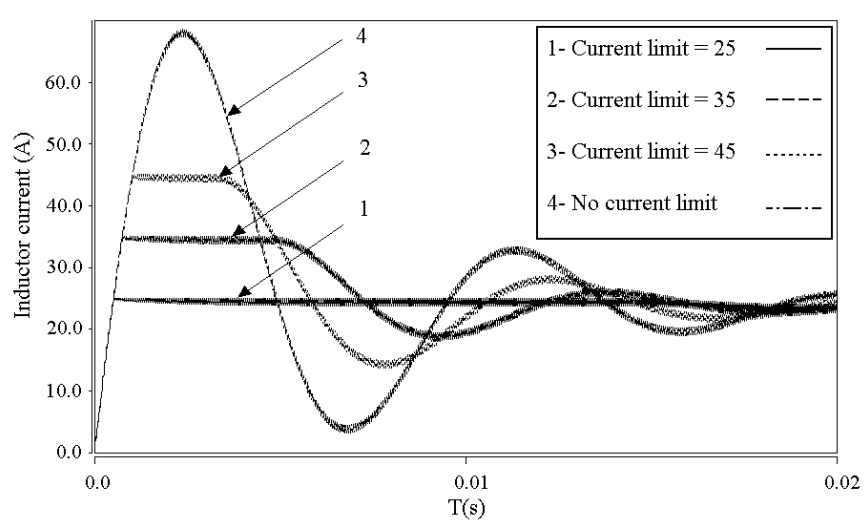

Fig. 6. Simulation results of inductor current of a buck converter for various values of current limit.

The effect of the current limiter is the same as with the buck converter. However, for the boost converter the inductor current exceeds the current limit at the beginning. The reason for this is as follows. In a boost converter when the output voltage is less than the input voltage, turning the switch off cannot decrease the inductor current, because the voltage on the inductor is still positive. In the steady state the inductor current cannot exceed the current limit. It is clear that a current limit should be chosen that is larger than the steady state value of the inductor current. If a current limit is chosen that is very near to the steady state value of the inductor current, the response will be sluggish.

In both simulations, it can be seen that at the beginning, the current mode controller governs the converter. However, at the steady state, the one-cycle controller takes over its duty. In Fig. 9 , this matter is shown. We can see that at the very beginning, when the current is less than the current limit value, the onecycle controller produces the reset command. Then the current reaches the limit and the current limit controller produces the reset command. Some time later when the current returns to its normal range the one-cycle controller completely governs at the steady state.

\section{EXPERIMENTAL VERIFICATION}

A buck converter was built to prove the theoretical and simulation results of the control method. The converter parameters

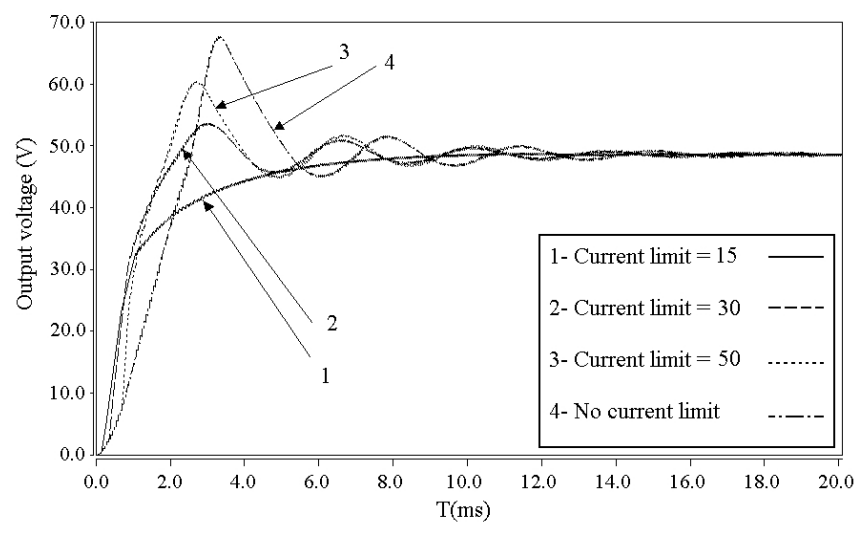

Fig. 7. Simulation results of output voltage of a boost converter due to various values of current limit.

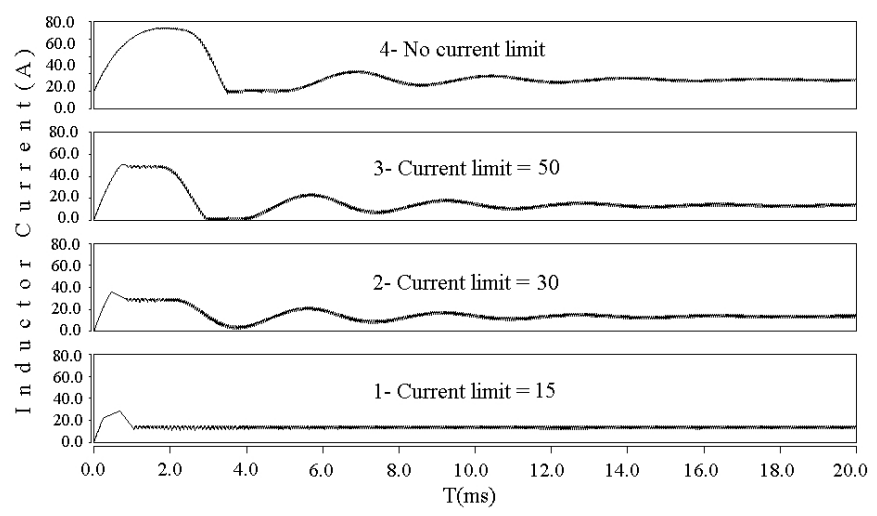

Fig. 8. Simulation results of inductor current of a boost converter for various values of current limit.
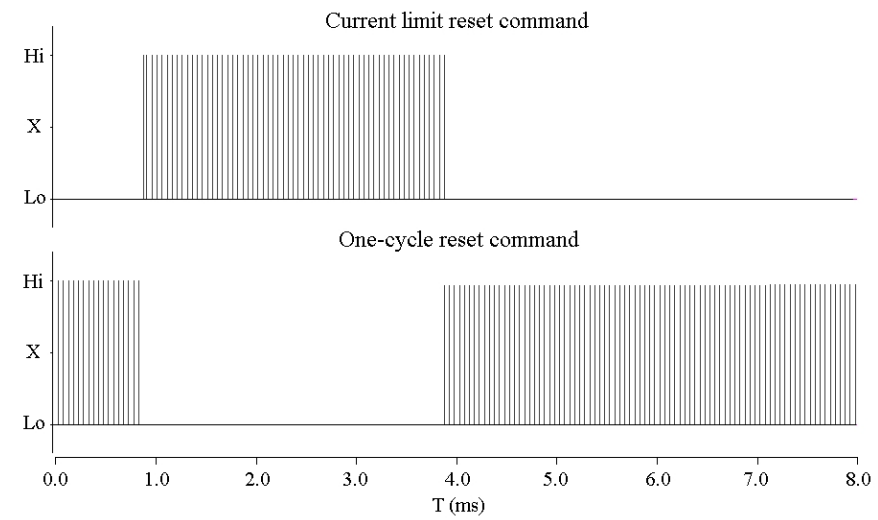

Fig. 9. Simulation results of the reset command of one-cycle controller and current limit controller for buck converter.

are as follows:

$L=0.5 m H, r_{L}=0.3 \Omega, C=470 \mu F, R=8 \Omega, V_{g}=$ $30 \mathrm{~V}, V_{0}=15$, and $f=25 \mathrm{kHz}$.

The results completely support the simulations. In Fig. 10 , the step response of the one-cycle controller has been shown. As can be seen, although the step response has a large overshoot, OCC has completely rejected the line variations. In Fig. 11, the step response of the combined one-cycle and current limit controllers for a specific value of the current limit has been shown. In this case, by selecting a current limit that is 


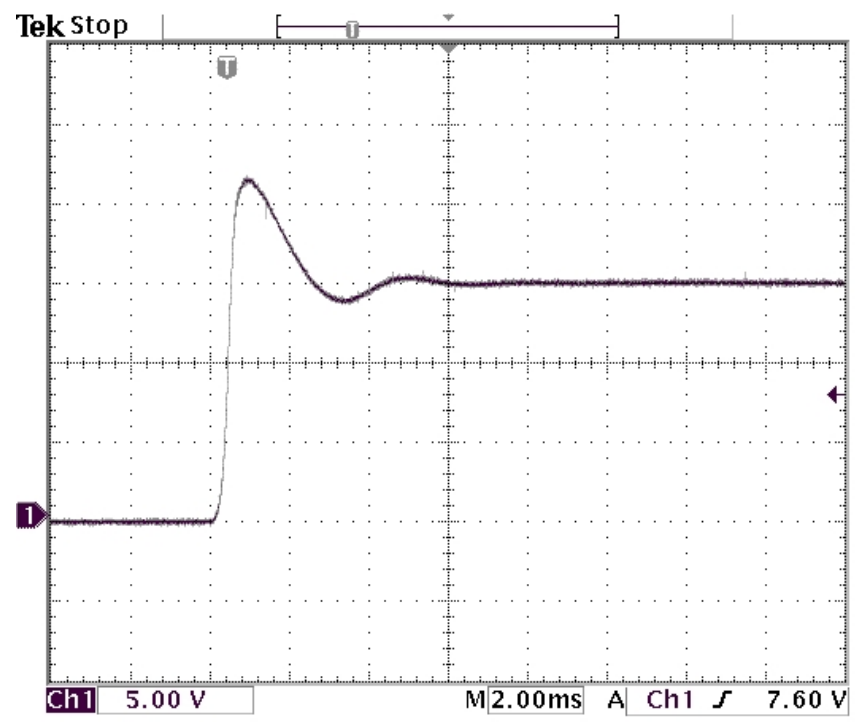

Fig. 10. Step response of one-cycle controller; experimental result.

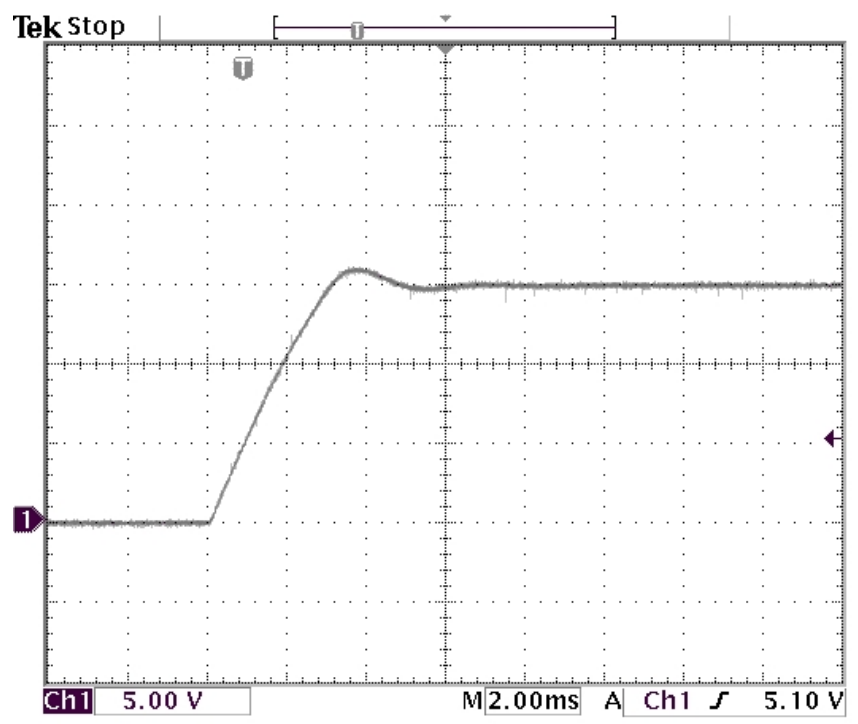

Fig. 11. Step response of the combined method for a given value of current limit; experimental result.

near the steady state value of the inductor current, the response has become nearly damped and the overshoot has decreased. On the other hand, the line disturbance characteristic of OCC has remained unchanged. Furthermore, it is possible to increase the current limit value to get a faster response.

In Fig. 12 the output voltage and in Fig. 13, the inductor current for different values of the current limit are given. The current limit has been set by a potentiometer and it varies between $2 \mathrm{~A}$ to $10 \mathrm{~A}$. By increasing the value of the current limit, the inductor current and the output voltage waveforms reach the conventional OCC response. On the other hand, by decreasing the current limit value, the overshot on the current and voltage decrease. Therefore, it is possible to compromise between the overshoot and the settling time of the controller and there is enough flexibility to get a faster or a lower overshoot response. It is known that in a conventional onecycle controller this feature is not available.

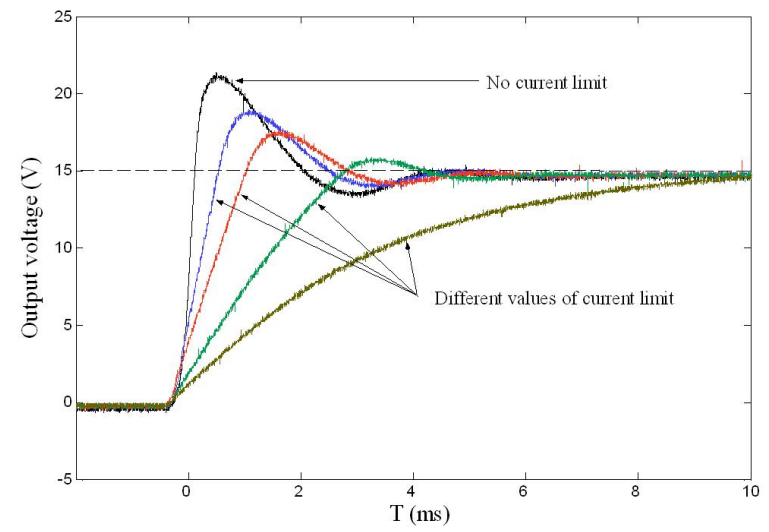

Fig. 12. Step response of the combined method due to different values of current limit; experimental results.

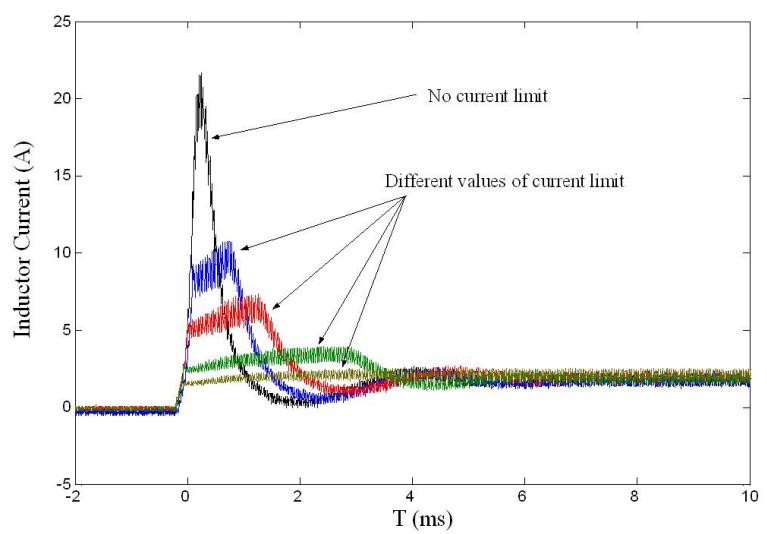

Fig. 13. Inductor current of the combined method due to different values of current limit; experimental results.

\section{CONCLUSIONS}

The most important feature of OCC is its great line disturbance rejection capability which in this matter is more powerful than conventional PID controllers. On the other hand, conventional one-cycle controllers in many cases have a large output overshoot. This output voltage overshoot is undesirable and in many applications it is hazardous. As a result it limits the application of one-cycle controllers. In this paper a method for the combination of a one-cycle controller and a current mode controller is given. It is shown that for one-cycle controlled buck and boost converters this method improves the transient characteristic. The main idea is to limit the overshoot of the inductor current at the transient to prevent the output from oscillation.

It is clear that by this combined method, the usual problem of peak current mode control instability will not occur, because the current mode controller works for a short period at the transient time.

The proposed method limits stress on the converter components and offers the overload protection of current mode control. It also improves the transient response and has the advantage of line ripple rejection of a one-cycle controller. A prototype buck converter was built to prove the capability of 
the method. The circuit of the combined controller when compared to that of a conventional one-cycle controller has just few extra elements and it is easy to build. The experimental results agree with the simulations and show that the method is practical. On the other hand, the necessity of a current limiter in the practical converters is a matter of choice for the designer. Hence, this method does not impose unnecessary costs but gives more flexibility to the designer to improve the transient response of the OCC method, as it is desired.

\section{REFERENCES}

[1] K. Smedley and S. Cuk, "One-cycle control of switching converters," IEEE Trans. power Electron., Vol. 10, pp. 625- 633, 1995.

[2] K. M. Smedley, L. Zhou, and C. Qiao, "Unified constant frequency integration control of active power filters steady state and dynamics," IEEE Trans. Power Electron., Vol. 16, pp. 369-375. 2001.

[3] C. Qiao, and K. M. Smedley, "Unified constant frequency integration control of three phase standard bridge boost rectifiers with power factor correction," IEEE Trans. Ind. Electron. , Vol. 50, pp. 100-107, 2003.

[4] D. Ma, W. H. Ki, and C. Y. Tsui, "An Integrated One-Cycle Control Buck Converter with Adaptive Output and Dual Loops for Output Error Correction," IEEE Journal of solid state circuits, Vol. 39, pp. 140-149, 2004.

[5] F. D. Tan, and R. D. Middlebrook, "A unified model for currentprogrammed converters," IEEE Trans. Power Electron., Vol. 10, pp. $397-$ 408, 2001.

[6] T. Suntio, "Analysis and modeling of peak-current mode controlled buck converter in DICM," IEEE Trans. Ind. Electron., Vol. 48, pp. 127-135, 2001.

[7] L. Dixon, "Average current mode control of switching power supplies," Unitrode power supply design seminar manual, pp. 356-369, 1990.

[8] E. Santi, and S. Cuk, "Modeling of one-cycle controlled switching converters," in Proc INTELEC, 92., 14th Int. conf., Washington DC, pp. 131-138, 1992.

[9] M. Ruzbehani, L. Zhou, M. Wang, "A new approach in combining onecycle controller and PID controller," in Proc of 2004 IEEE International Symposium on Industrial Electronics., Ajaccio, France, pp. 1173-1177, 2004.
[10] M. Ruzbehani, L. Zhou, M. Wang, "Use of a nonlinear controller to improve one-cycle controller response," Journal of applied science, Vol. 6, No. 6, pp. 1343-1350, 2006.

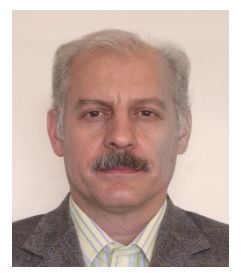

Mohsen Ruzbehani received his B.S., M.S. and Ph.D. in Electrical Engineering from the Isfahan University of Technology, Isfahan, Iran, in 1989, Shiraz University, Shiraz, Iran, in 1996 and Chongqing University, Chongqing, China in 2004 respectively. He has been with the Laser \& Optics Research School, Tehran, Iran, formerly known as the Laser Research Center, since 1996 where he is currently an Assistant Professor and Vice President in Research and Education. His research interest include, control methods in power electronics, chaos control and laser electronics.

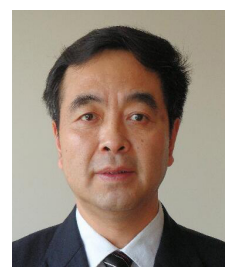

Luowei Zhou received his B.S., M.S. and Ph.D. in Electrical Engineering at Chongqing University, Chongqing, P.R. China, in 1982, 1988 and 2000, respectively. Since 1982, he has been with the College of Electrical Engineering at Chongqing University where he is now a full professor. He was a visiting professor at the University of California, Irvine, between September 1998 and August 1999. He is the administrative director of the China Society of Power Supply. His major fields of interest include the analysis and control of power electronics circuits, the realization of active power filters, power factor correction techniques and high frequency power conversion. He has published more than 60 papers, holds one US and three Chinese patents, and has three patents pending.

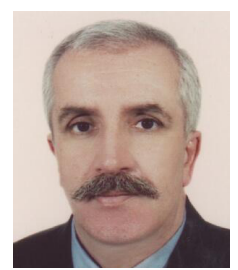

Nasser Mirzaei received his M.S. degree in Electronics Engineering from the Polytechnic of Turin, Italy, in 1986. Since then, he has been with the Laser \& Optics Research School, Tehran, Iran, formerly known as the Laser Research Center, where he is currently the head of the Semiconductor Lasers Research Group. His research interests include power electronics, industrial control and crystal growth engineering. 